\title{
An Equivalent-control Quantization Sliding Mode Controller for a Buck Converter
}

\author{
Qianqian $\mathrm{Li}^{1, \mathrm{a}}$, Peter $\mathrm{Liu}^{2, \mathrm{~b}}$ and Yisong Wang ${ }^{1, \mathrm{c},{ }^{*}}$ \\ ${ }^{1}$ Department of Computer Science and Technology, Guizhou University, Guiyang, China \\ ${ }^{2}$ Department of Electrical Engineering, Tamkang University, Taipei, Taiwan \\ a819169403@qq.com, bpliu@mail.tku.edu.tw, cyswang@gzu.edu.cn
}

Keywords: equivalent-control, quantizer, sliding mode voltage control, DC/DC power system.

\begin{abstract}
This paper studies the DC-DC buck converter response controlled by two second-order single-input control techniques: equivalent-control quantization sliding mode voltage control (QSMVC) and general sliding mode voltage control (SMVC). Simulations illustrate the behaviors of the equivalent-control based SMVC system under uniform and logarithmic quantized state feedback. The output voltage and inductor current of both models were studied and compared under normal conditions, load step change, load linear variation, and input voltage step change. It shows that the equivalent-control QSMVC system performs better than the general SMVC system in terms of robustness and stability. The equivalent-control QSMVC system prevents the sliding mode controller from operating at a frequency that is too high for the power switch to respond. Also, by setting the value of quantization parameters the output voltage value can be greater than $5.999 \mathrm{~V}$ with less than $0.17 \%$ deviation from $6 \mathrm{~V}$.
\end{abstract}

\section{Introduction}

Switching power supply control applications have been extensively studied, many controllers have been developed to control switching-mode power supplies (SMPSs) and to solve problems regarding non-linear components in the converter structure, line and load variations, and electro-magnetic interferences (EMI) [1], some paper are [2] [3] [4] [5]. See [1] for discussion the advantages and drawbacks of each control method, and under which conditions different control methods are suitable. Sliding mode (SM) controllers are well known for their robustness and stability for solving these problems. One of the most salient features of the sliding mode scheme in a variable structure system (VSS) is the ability to achieve a response independent of the parameter of the system; the only constraint is the canonical form description of the system.

The sliding mode is a nonlinear control that conforms to the nonlinear structure of the switch mode power supply, and it provides a means of implementing a control action that utilizes the inherently variable structure characteristics of the DC-DC converter. Due to the advantages of SMC, has been widely studied and implemented, with a key focus being quantization feedback. Uniform and logarithmic quantizers, which can make the system states converge into a band by setting the quantization parameters to control the width of the band, are most commonly used. See [6] [7] for further detail on quantization problems in SMC. Quantization behaviors in Equivalent-control based SMC systems has been mentioned in [7] and it is understood that has not been used on buck converter. In this paper, to prove its good performance, we made its behaviors compare with the behaviors of general sliding mode control which is described in [8].

This paper is organized as follows. Section 2 introduces some preliminaries including the buck converter, equivalent-control of sliding mode voltage control, and quantizer function. Section 3 details the equivalent-control based on sliding mode voltage control systems with quantizer (equivalent-control QSMVC). Section 4 presents the experimental results of a buck converter controlled by the equivalent-control QSMVC or by general SMVC. Finally, Section 5 summarizes our findings. 


\section{Preliminaries}

This section introduces some basic concepts, including two commonly used quantizers, and the theoretical aspects of the modulated ramp Pulse-Width-Modulation (PWM) [9] generator applied in the equivalent-control sliding mode voltage control of buck converter systems. We assume that everyone knows how the buck converter works and does not elaborate.

\subsection{Equivalent-control Based on a Sliding Mode Voltage Control}

The sliding mode is a variable structure system (VSS), the instantaneous states of the system determine that a change occurs and force the system trajectory to maintain a suitable selected surface called a sliding surface. However, the important task is to find the state variables and select the appropriate sliding surface. Let $\beta=\mathrm{R} 2 /(\mathrm{R} 1+\mathrm{R} 2)$ be partial output voltage ratio, $V_{r e f}$ and $\beta V_{o}$ be the reference and sensed output voltage, and $u \in\{1,0\}$ be the switching state of power switch $S_{W}$ as 'ON' or 'OFF'. The output voltage error $x_{1}$, the rate of voltage error change $x_{2}$, and the integral of voltage error $X_{3}$ are state variables expressed as

$$
\begin{aligned}
& x_{1}=V_{\text {ref }}-\beta V_{o} \\
& x_{2}=\dot{x}_{1}=\frac{\beta}{C}\left(\frac{V_{o}}{R_{L}}-\int \frac{u V_{i n}-V_{o}}{L} d t\right) \\
& x_{3}=\int x_{1} d t
\end{aligned}
$$

From Eq. (1), the system state space model can be derived as:

$$
\dot{x}=A x+B u+D
$$

where

$$
\begin{aligned}
A & =\left[\begin{array}{ccc}
0 & 1 & 0 \\
-a_{1} & -a_{2} & 0 \\
1 & 0 & 0
\end{array}\right], B=\left[\begin{array}{c}
0 \\
a_{3} \\
0
\end{array}\right], D=\left[\begin{array}{c}
0 \\
a_{4} \\
0
\end{array}\right], x=\left[\begin{array}{c}
x_{1} \\
x_{2} \\
x_{3}
\end{array}\right], x \in R^{3}, a_{1}=\frac{1}{L C}, a_{2}=\frac{1}{R_{L} C}, \\
a_{3} & =-\beta \frac{V_{\text {in }}}{L C}, a_{4}=\frac{V_{r e f}}{L C} .
\end{aligned}
$$

The sliding surface is described as

$$
S=c_{1} x_{1}+c_{2} x_{2}+c_{3} x_{3}=J x
$$

where $J=\left[\begin{array}{lll}c_{1} & c_{2} & c_{3}\end{array}\right]$, and $c_{1}, c_{2}$, and $c_{3}$ represent the control parameters as sliding coefficients. To set the coefficients of the sliding surface, the system can consider its required bandwidth $f_{B W}[8]$.

To ensure that a system follows its sliding surface, a control law must be obeyed, and to prevent the sliding mode controller from operating at a too high frequency, a hysteresis band is introduced in [10]. Suppose that $-\xi \leq S \leq \xi$. In $-\xi \leq S \leq \xi, S_{W}$ remains in its previous state. The value of $\xi>0$ is determined by the choice of sliding mode. Then, under ideal sliding mode conditions, the control law is defined as

$$
u=\left\{\begin{array}{l}
1, S>\xi \\
0, S<-\xi
\end{array}\right.
$$

Using the Lyapunov's Method [11] to ensure that SMC is realizable in this system, an existence condition [12] $\dot{V}=S \dot{S}<0$ must be obeyed.

When the system enters the sliding phase, by incorporating the hysteresis band, it is not difficult to find that at the transition point of $S= \pm \xi$, there exists a corresponding point $\dot{S}=\mp \alpha$. Further, by combining Eqs.(2) and (3) and the time derivative of (3), the condition can be transformed into $S \dot{S}=S(J A x+J B u+J D)<0$. Therefore, we can get 


$$
\left\{\begin{array}{l}
J A x+J B+J D+\alpha \operatorname{sgn}(S)<0, S>\xi ; \\
J A x+J D+\alpha \operatorname{sgn}(S)>0, S<-\xi .
\end{array}\right.
$$

Where $\alpha>0$ is the same that in $u_{s}$ introduced in the next paper. Obviously, if this condition is met, the above conditions $S \dot{S}<0$ must be satisfied. We obtain the inequality

$$
J A x+J B+J D+\alpha \operatorname{sgn}(S)<0<J A x+J D+\alpha \operatorname{sgn}(S) .
$$

Here, the inequality can be translated into

$$
0<c_{3} X_{1}+\left(c_{1}-\frac{c_{2}}{R_{L} C}\right)_{X_{2}}+\frac{c_{2}}{L C} \beta V_{0}+\alpha \operatorname{sgn}(S)<c_{2} \frac{\beta V_{i n}}{L C}
$$

or rearranged as

$$
f=\frac{1}{c_{2} a_{3}}\left[\left(a_{1} c_{2}-c_{3}\right)_{X_{1}}+\left(a_{2} c_{2}-c_{1}\right)_{X_{2}}-a_{4} c_{2}\right]-\frac{1}{c_{2} a_{3}} \alpha \operatorname{sgn}(S),(0<f<1) .
$$

The equivalent-control based on SMVC is

$$
u^{*}=u_{e q}+u_{s}
$$

Where the solution of the controller consists of two parts, a continuous equivalent control part is $u_{e q}$ and a discontinuous control part is $u_{s}$, which are the outputs of an equivalent-control SMC system [13]. Many studies use $u_{e q}$ as the output of the SMC system, which called the general SMC system [8]. The equivalent control $u_{e q}$ is the amount of control applied by the sliding mode controller when the system enters the sliding mode dynamics $S=0$. The equivalent control $u_{e q}$ is obtained by taking Eq. (2) into $\dot{S}=0$, where $J B$ is non-singular, as follows:

$$
\begin{aligned}
u_{e q} & =-(J B)^{-1} J(A x+D) \\
& =\frac{1}{c_{2} a_{3}}\left[\left(a_{1} c_{2}-c_{3}\right)_{X_{1}}+\left(a_{2} c_{2}-c_{1}\right)_{X_{2}}-a_{4} c_{2}\right] .
\end{aligned}
$$

The discontinuous control part $u_{s}$ is used to overcome the uncertainty of the system and to give the system new features as follows:

$$
u_{s}=-(J B)^{-1} \alpha \operatorname{sgn}(S)=-\frac{1}{c_{2} a_{3}} \alpha \operatorname{sgn}(S) .
$$

Where $\alpha>0$ is a controller parameter, $\operatorname{sgn}(x)$ is a signum function where $\operatorname{sgn}(x)=1$ for $x>0, \operatorname{sgn}(x)=-1$ for $x<0$, and $\operatorname{sgn}(x)=0$ for $x=0$. Since $0<u^{*}<1$. In the PWM control system[8], the duty cycle $d$ is expressed as $d=V_{c} / \hat{V}_{\text {ramp }}$, where $0<d<1$, and $V_{c}$ and $V_{\text {ramp }}$ represent the control signal of the PWM and the peak magnitude ramp signal, respectively. Drawing on ideas in [8] [14], we obtain $V_{c}=u^{*}$ and $\hat{V}_{\text {ramp }}=1$.

\subsection{Quantizer}

Here, we will introduce two commonly used quantizers: uniform quantizer and logarithmic quantizer.

\subsubsection{Uniform Quantizer}

A uniform quantizer with quantizing level $q$ can be defined as

$$
Q_{\text {uni }}(x)=q \times \operatorname{round}\left(\frac{x}{q}\right)
$$


where $Q_{u n i}(x)$ is the quantization operator, and round $($.$) is a function that rounds x$ value to the nearest integer value. For example, round $(1.2)=1$ and $\operatorname{round}(1.6)=2$. This can be written in more detail as

$$
Q_{u n i}(x)=\left\{\begin{array}{l}
k q, \text { if } x \in\left[\frac{2 k-1}{2} q, \frac{2 k+1}{2} q\right], k \in N^{+} ; \\
0, \text { if } x \in\left(-\frac{1}{2} q, \frac{1}{2} q\right) ; \\
-k q, \text { if } x \in\left[-\frac{2 k+1}{2} q,-\frac{2 k-1}{2} q\right], k \in N^{+} .
\end{array}\right.
$$

The quantization error of a uniform quantizer can be expressed as

$$
e(x)=Q_{\text {uni }}(x)-(x)
$$

, where $|e| \leq \frac{q}{2}$, and the quantization level $q$ is a constant. The maximum rounding error is half of $q$. The smaller $q$ value is, the closer the quantitative result to the true value will be. The area of $x \in\left(-\frac{1}{2} q, \frac{1}{2} q\right)$ is called the dead-zone area, and it causes the system to converge and chatter around the area of $Q_{u n i}(x)=0$.

\subsubsection{Logarithmic Quantizer}

The quantization level of a logarithmic quantizer is the variable described by

$$
l=\left\{\rho^{i} \mu: i=0, \pm 1, \pm 2, \ldots\right\} \cup\{0\}
$$

where $\mu>0$ is a scaling parameter and $\rho \in(0,1)$ is quantization density. A small $\rho$ implies coarse quantization, whereas a large $\rho$ implies dense quantization. $i<r$, where $r \in N^{+}$is a constant representing the number of system quantization steps.

The logarithmic quantization operator $Q_{\log }(x)$ is

$$
Q_{\log }(x)=\left\{\begin{array}{l}
\rho^{i} \mu, \text { if } \frac{1}{1+\sigma} \rho^{i} \mu<x<\frac{1}{1-\sigma} \rho^{i} \mu \\
0, \text { if }-\frac{1}{1+\sigma} \rho^{r} \mu<x<\frac{1}{1+\sigma} \rho^{r} \mu \\
-\rho^{i} \mu, \text { if }-\frac{1}{1-\sigma} \rho^{i} \mu<x<\frac{1}{1+\sigma} \rho^{i} \mu .
\end{array}\right.
$$

, and quantization error is expressed as

$$
e(x)=Q_{\log }(x)-x
$$

where $\sigma=\frac{1-\rho}{1+\rho}$, and $\sigma$ represents the maximum relative error of the logarithmic quantized variable, $|e| \leq \sigma|x|$.

The logarithmic quantizer has a dead-zone area $x \in\left\{-\frac{1}{1+\sigma} \rho^{r} \mu, \frac{1}{1+\sigma} \rho^{r} \mu\right\}$. This dead-zone area is controlled by $r$, and it can be minimized by choosing a suitable integer $r$. For the stability problem of the control process, the logarithmic quantizer is more efficient than the uniform quantizer. The logarithmic quantizer can faster converge, and has smaller dead-zone area than the uniform quantizer. 


\section{The SMVC System with Quantized State Feedback}

This section introduces the problems relating to the SMVC system with quantized state feedback. It is assumed that the input variables to the sliding mode controller are simply the quantized value of the system states. The quantizer is present between the system states output and the sliding mode controller input as shown in Figure 1. In Figure 1, the system states pass through the quantizer and are quantized as $\hat{X}=Q(x)$, where the quantized value of $x$ is represented by a symbol $\hat{X}$ and called quantization states. The symbols $\hat{u}$ and $\hat{S}$ represent the output of the controller and the switching function, respectively. Therefore, the mathematical model of the sliding mode control system Eqs.(2), (3), (9) can be rewritten as:

$$
\begin{gathered}
\dot{x}_{1}=x_{2} \\
\dot{x}_{2}=-a_{1} x_{1}-a_{2} x_{2}+a_{3} \hat{u}^{*}+a_{4} \\
\dot{x}_{3}=x_{1} \\
\hat{S}=c_{1} \hat{x}_{1}+c_{2} \hat{X}_{2}+c_{3} \hat{x}_{3}=J \hat{x} \\
\hat{u}^{*}=\hat{u}_{e q}+\hat{u}_{s}
\end{gathered}
$$

$\dot{X}_{1}$ and $\dot{X}_{3}$ are not adjusted, and the quantizations $\hat{u}_{e q}, \hat{u}_{s}$, and $\dot{X}_{2}$ are adjusted as:

$$
\begin{aligned}
\hat{u}_{e q} & =-(J B)^{-1} J(A \hat{x}+D) \\
& =\frac{1}{c_{2} a_{3}}\left[\left(a_{1} c_{2}-c_{3}\right) \hat{X}_{1}+\left(a_{2} c_{2}-c_{1}\right) \hat{X}_{2}-a_{4} c_{2}\right] \\
\hat{u}_{S} & =-(J B)^{-1} \alpha \operatorname{sgn}(\hat{S})=-\frac{1}{c_{2} a_{3}} \alpha \operatorname{sgn}(\hat{S})
\end{aligned}
$$

, where $e_{1}$ and $e_{2}$ are quantization errors of $x_{1}$ and $x_{2}$, respectively.

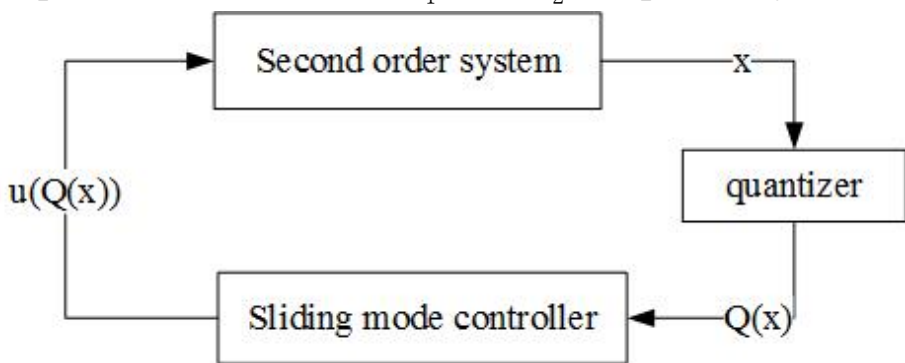

Figure 1. The SMC system with quantized state feedback.

As be defined in [7], the SMC with quantized state feedback is called a quantized sliding mode control (QSMC), and the sliding mode in QSMC is called a quantized sliding mode (QSM). In this paper the QSMC system is characterized by Eqs.(18), (19), and (20), where QSM is the quantized switching function Eq.(19). With the role of the quantizer, the system can become a hybrid system [15].

To make the system achieve a steady state after sliding on the switching surface, the condition, as derived from Eqs.(5) and (23) is

$$
\alpha \geq\left|\left(a_{1} c_{2}-c_{3}\right) e_{1 \max }\right|+\left|\left(a_{2} c_{2}-c_{1}\right) e_{2 \max }\right|,
$$

where $e_{1 \max }$ and $e_{2 \max }$ are the maximum quantization errors of $x_{1}$ and $x_{2}$. The derivation steps are $S \dot{S}=S\left(c_{1} \dot{X}_{1}+c_{2} \dot{X}_{2}+c_{3} \dot{X}_{3}\right)<0$. Substituting Eqs.(18), (23) to it, we can get

$$
S\left(\left(a_{1} c_{2}-c_{3}\right) e_{1}+\left(a_{2} c_{2}-c_{1}\right) e_{2}\right)<\alpha|S| \text {. }
$$

Then, the inequality $\alpha \geq\left|\left(a_{1} c_{2}-c_{3}\right) e_{1 \max }\right|+\left|\left(a_{2} c_{2}-c_{1}\right) e_{2 \max }\right|$ can be obtained. 


\section{System Simulation and Analysis}

To compare the performance of equivalent-control QSMVC and general SMVC, simulations using a benchmark converter with the specifications given in Table 1 were carried out in MATLAB $\backslash$ Simulation ${ }^{\mathrm{TM}}$.

Table 1. Specification of buck converter.

\begin{tabular}{|c|c|c|}
\hline Description & Parameter & Nominal Value \\
\hline Input voltage & Vin & $12 \mathrm{~V}$ \\
\hline Inductance & $\mathrm{L}$ & $180 \mathrm{mH}$ \\
\hline Capacitance & $\mathrm{C}$ & $100 \mu \mathrm{F}$ \\
\hline Load resistance & $\mathrm{RL}$ & $3 \Omega$ \\
\hline Switching frequency & $\mathrm{fS}$ & $200 \mathrm{kHz}$ \\
\hline Target output & Vod & $6 \mathrm{~V}$ \\
\hline Reference voltage & Vref & $3 \mathrm{~V}$ \\
\hline Partial voltage ratio & $\beta$ & 0.5 \\
\hline
\end{tabular}

In the equivalent-control QSMVC system, let the system state $x_{2}$ pass through the uniform quantizer. As $X_{2}$ is the rate of voltage error change, its minimum value is relatively large compared with $\rho^{r} \mu$. In contrast, if we choose logarithmic quantizer, the state will never enter the dead-zone domain. Let the system states $x_{1}, x_{3}$ pass through the logarithmic quantizer. As the value of $x_{1}$ and $X_{3}$ are little, the dead-zone area of logarithmic quantizer can be controlled as small as possible by selecting suitable $r$ value. Other values of the simulation parameters used in simulation are $q=10$, $\rho=0.8, \mu=0.1$, and $r=25$.

\subsection{Normal Condition}

First, we consider the steady state under normal conditions, that is, the input voltage and load resistance are not changed. The simulation results of the output voltage and inductor current controlled by the equivalent-control QSMVC system and general SMVC system are shown in Figure 2 and Figure 3. The output voltage and inductor current fluctuation intervals are $(5.991 \mathrm{~V}, 5.992 \mathrm{~V})$ and $(1.95 \mathrm{~A}, 2.05 \mathrm{~A})$, respectively. For the equivalent-control QSMVC system, by setting the value of quantization parameters $r, \rho, \mu, q$, the output voltage and inductor current are more stable and approach a straight line. In fact, the output voltage value is bigger than $5.999 \mathrm{~V}$ with less than $0.17 \%$ deviation from $6 \mathrm{~V}$, and the inductor current interval is $(2 \mathrm{~A}, 2.01 \mathrm{~A})$ located in the middle of the inductor current waveform controlled by the general SMVC system.

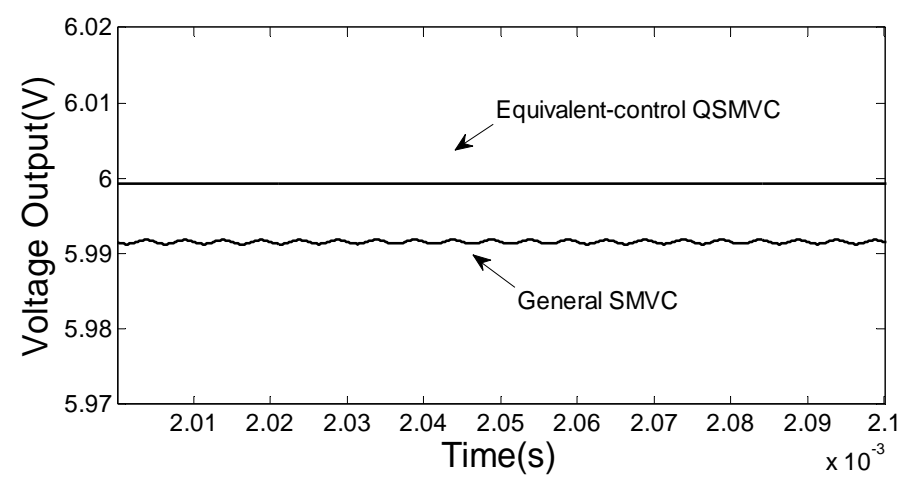

Figure 2. The steady-state output voltage under normal conditions. 


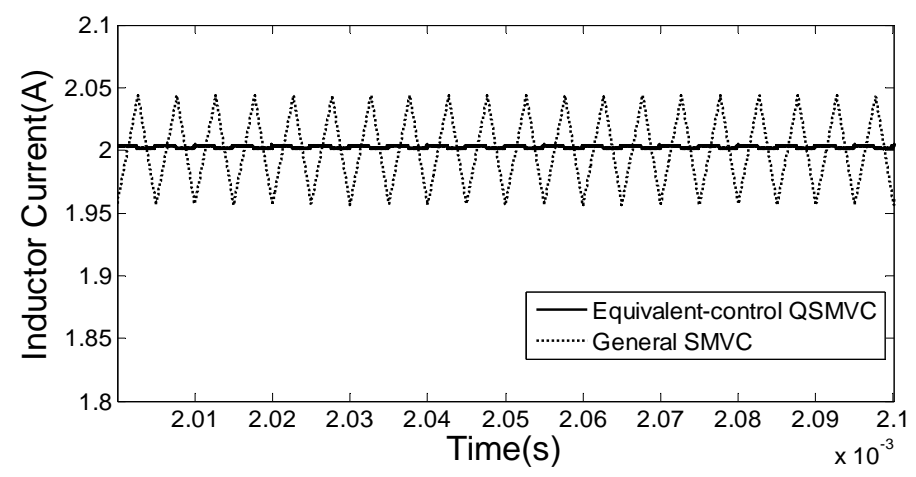

Figure 3. The steady-state inductor current under normal conditions.

The next step is to verify that the equivalent-control QSMVC system has robustness and stability.

\subsection{Load Step Change}

We start our simulation with load $\mathrm{R}_{\mathrm{L}}=3 \Omega$, then apply a step change to $24 \Omega$ at time $=0.005 \mathrm{~s}$. The simulation results in Figure 4, and 5 show the output voltage and inductor current change process controlled by the two control systems from the steady state before the load change to the steady state after the load change. As illustrated in Figure 4, when the load resistance step changes, the output voltage of both control systems has an overshoot ripple change with amplitude about $0.4 \mathrm{~V}$. From the enlarged part of Figure 4 that the equivalent-control QSMVC system reaches steady state earlier than the general SMVC system. We also find that the steady-state output voltage in equivalent-control QSMVC system before the load step change is the same as the steady-state output voltage after the load step change, while general SMVC system shows a slight offset. For this phenomenon, we do not give figures. Comparing the output voltage of the two systems, it can be concluded that QSMVC has better robustness and stability regarding load step change. Under the two control systems, when the resistance step changes, the inductor current drops rapidly and reaches steady state quickly, and the overall trend of the inductor current is the same. The change process of the inductor current shows is shown in Figure 5.

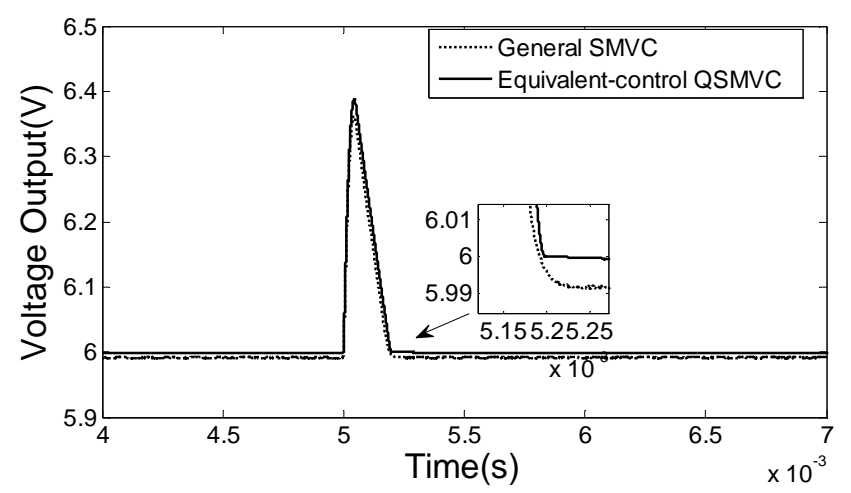

Figure 4 . The voltage output change process in the case of step change in load at time $=0.005 \mathrm{~s}$.

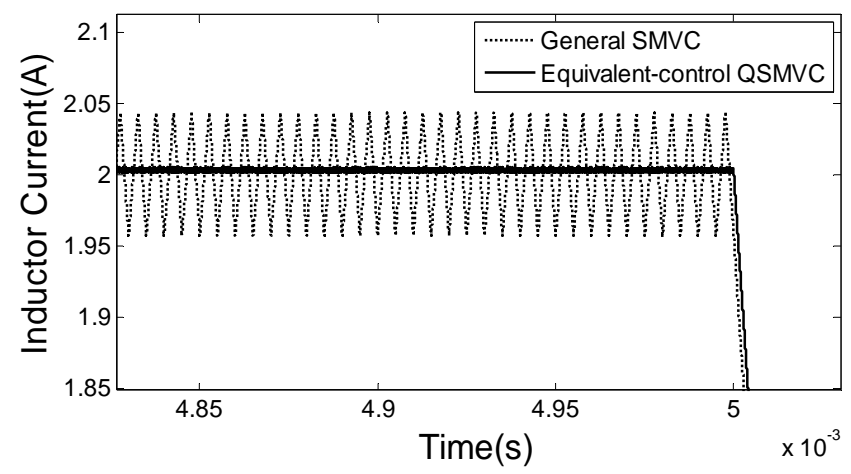

(a) The inductor current before step change. 


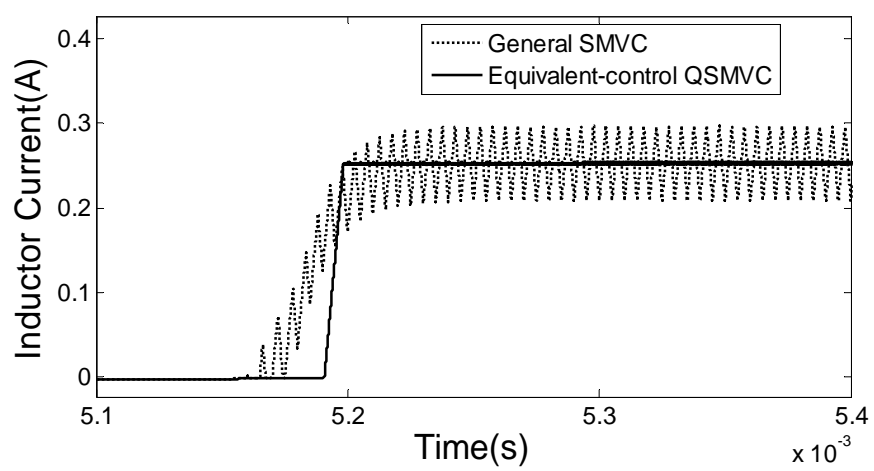

(b) The inductor current after step change.

Figure 5. The inductor current change process in the case of step change in load at time $=0.005 \mathrm{~s}$.

Figure 5(a) and Figure 5(b) are the enlarged views of the load step before and after the step change, respectively. In Figure 5(b), it can be clearly seen that the current drop recovers more quickly in the equivalent-control QSMVC when the step load change is applied. Further, the inductor current value is close to a straight line, and is located in the middle of inductor current waveform controlled by the general SMVC system.

\subsection{Load Linear Change}

Figure 6 and 7 show output voltage and inductor current with load resistances linear change between $3 \Omega$ and $24 \Omega$ with the cycle time is $6.6 \times 10^{-3} \mathrm{~S}$. To be able to more clearly observe the changes in these two pictures, we cut a part of the pictures and zoom in Figure 8 and 9.

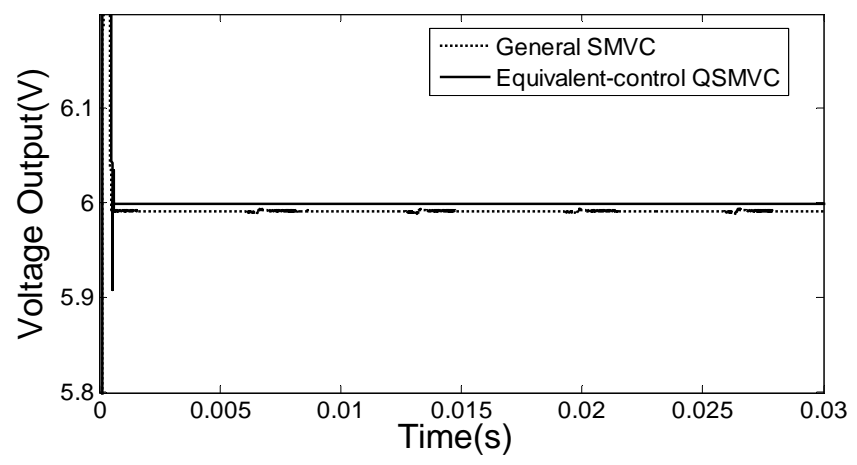

Figure 6 . The output voltage change process in the case of load periodic linear change.

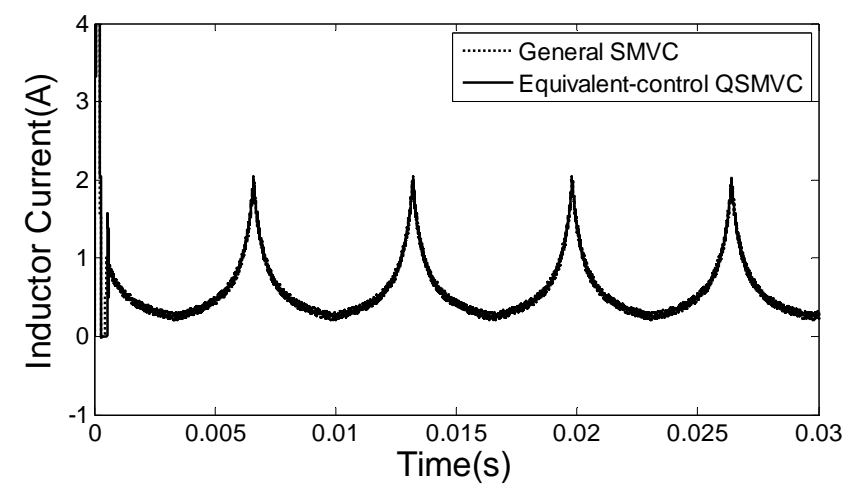

Figure 7. The inductor current change process in the case of load periodic linear change.

As illustrated in Figure 8, when the load undergoes a linear change, the output voltage of the equivalent-control QSMVC system is maintained in a straight line, and the output voltage of the general SMVC system has significant fluctuations. Figure 9 shows the variation of inductor current with load linear change. From the enlarged section, we see that regardless of the load value, the inductor current curve of the equivalent-control QSMVC system is located in the middle of the inductor current waveform controlled by the general SMVC system. time $=6.6 \times 10^{-3} \mathrm{~s}$ and time $=13.2 \times 10^{-3} \mathrm{~s}$ correspond to the resistance values of $3 \mathrm{~V}$ and $24 \mathrm{~V}$, respectively. The inductor current change trend of the two controllers is the same. With the resistance changes, the current first 
slowly down and then slowly increase, the graphics to time $=9.9 \times 10^{-3} \mathrm{~s}$ as the center of the form of symmetry between $6.6 \times 10^{-3} \mathrm{~s}$ and $13.2 \times 10^{-3} \mathrm{~s}$. The change of equivalent-control QSMVC is the curve change, while the general SMVC is the fluctuation change.

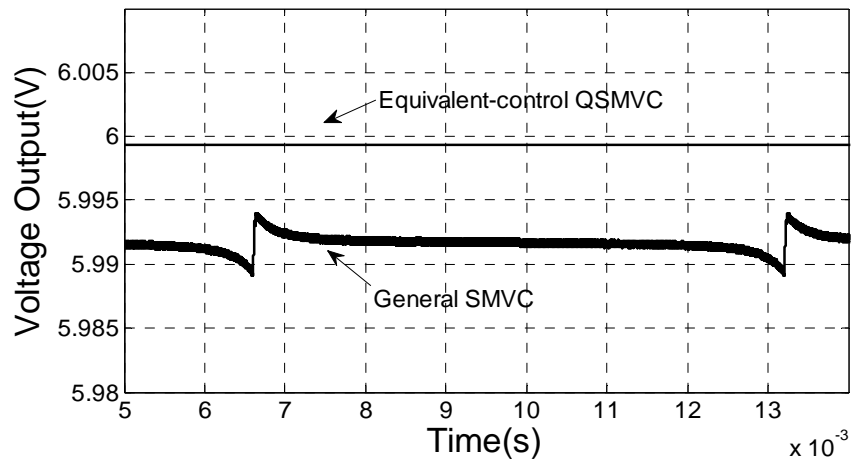

Figure 8 . The output voltage change process in the case of load periodic linear change.

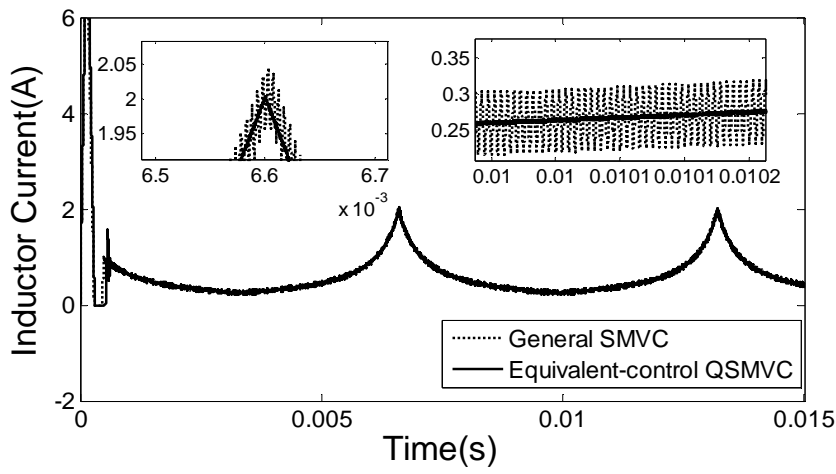

Figure 9. The inductor current change process in the case of load periodic linear change.

\subsection{Input Voltage Step Change}

In subsection 4.2 and 4.3, the changes of output voltage and inductor current in the two systems were described under load step change and linear change conditions. In this subsection, we discuss the changes in output voltage and inductor current under input voltage step changes. Our simulation starts with input voltage $V_{\text {in }}=12 \mathrm{~V}$, then applies a step change to $24 \mathrm{~V}$ at time $=0.005 \mathrm{~s}$. As illustrated in Figure 10, when the input voltage step changes, there is no change in the output voltage controlled by the equivalent-control QSMVC system. However, the output voltage controlled by the general SMVC system rapidly declines, and there is a clear gap between the stability values before and after the input voltage change. In Figure 11, before and after the input voltage step change, the inductor current controlled by the equivalent-control QSMVC is almost unchanged, while the amplitude of the inductor waveform is significantly larger in the general SMVC system. Comparing the two control systems, it can be concluded that QSMVC has better robustness and stability to input voltage step change.

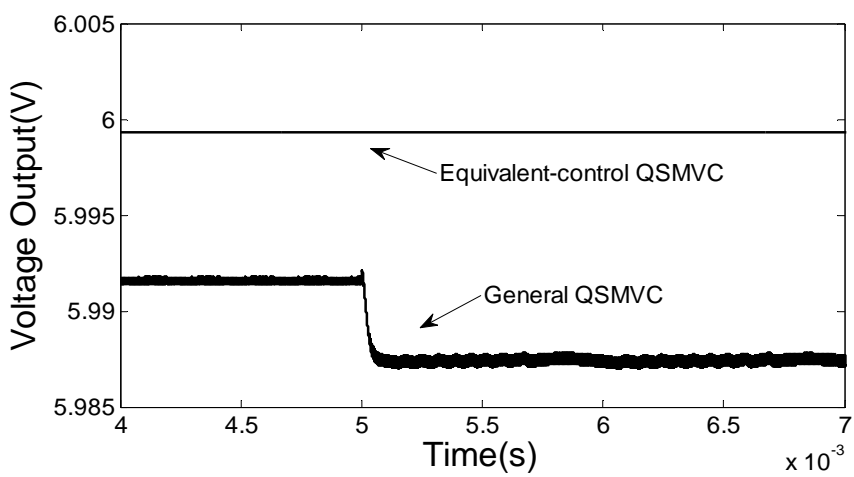

Figure 10. The output voltage change process in the case of step change in input voltage at time $=0.005 \mathrm{~s}$. 


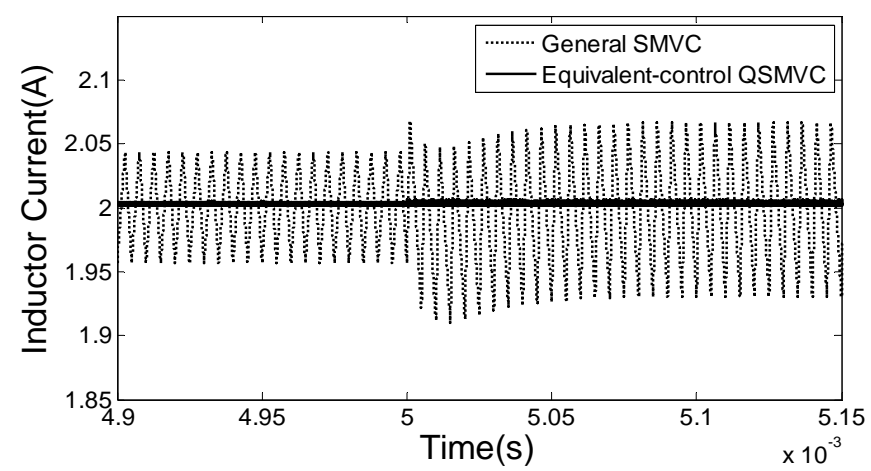

Figure 11. The inductor current change process in the case of step change in input voltage at time $=0.005 \mathrm{~s}$.

\section{Conclusion}

In this paper, a novel sliding mode QSM is introduced, and a uniform and logarithmic quantizer is used between the system state output and the control input. We focused on the effect of the buck converter controlled by an equivalent-control QSMVC by testing under normal conditions, load step change, load linear change, and input voltage step change, and compared the results with the buck converter controlled by a general SMVC.

\section{Acknowledgements}

We thank M. Ahmed, Vadim Ivanovich Utkin, Yan Yan, Xinghuo Yu and G. SPIAZZI for their insightful comments on the earlier version of the paper. This work is partially supported by NSFC under grant 63170161, Stadholder Fund of Guizhou Province under grant (2012)62, Outstanding Young Talent Training Fund of Guizhou Province under grant (2015)01 and and Science and Technology Fund of Guizhou Province under grant [2014]7640.

\section{References}

[1]. M.Ahmed, "Sliding mode control for switched mode power supplies," Acta Universitatis Lappeenrantaensis, 2004.

[2]. H. C. Lin and T. Y. Chang, "Analysis and design of a sliding mode controller for buck converters operating in dcm with adaptive hysteresis band control scheme," in International Conference on Power Electronics and Drive Systems, 2007, pp. 372-377.

[3]. R. W. Erickson and D. Maksimovic, "Current programmed control," 2001.

[4]. A. S. I. Zinober, Variable structure and Lyapunov control. Springer Berlin Heidelberg, 1994.

[5]. G. SPIAZZI, P. MATTAVELLI, L. ROSSETTO, and L. MALESANI, "Application of sliding mode control to switch-mode power supplies," Journal of Circuits System \& Computers, vol. 05, no. 3, pp. 337-354, 2011.

[6]. S. H. Yu and Y. H. Tsai, "Sliding-mode quantized control of a class-d audio power amplifier," in Power Electronics Conference, 2010, pp. 553 - 556.

[7]. Y. Yan and X. Yu, Quantization Behaviors in Equivalent-Control Based Sliding-Mode Control Systems. Berlin, Heidelberg: Springer Berlin Heidelberg, 2013, pp. 221-241.

[8]. S. Tan, Y. M. Lai, M. K. Cheung, and C. K. Tse, "A pulse-width-modulation based sliding mode controller for buck converters," in Power Electronics Specialists Conference, 2004. Pesc 04. 2004 IEEE, 2004, pp. 3647-3653 Vol.5.

[9]. S. A. A. Hussainy, R. G. Tandon, and S. Kumar, "Pwm based sliding mode control of dc-dc converters," in Advances in Power Conversion and Energy Technologies (APCET), 2012 International Conference on, Aug 2012, pp. 1-6. 
[10]. S. Tan, Y. M. Lai, C. K. Tse, and M. K. Cheung, "An adaptive sliding mode controller for buck converter in continuous conduction mode," in Applied Power Electronics Conference and Exposition, 2004. Apec '04. Nineteenth IEEE, 2004, pp. 1395-1400 Vol.3.

[11]. J. Slotine and W. Li, Applied nonlinear control. China Machine Press, 2004.

[12]. V. Utkin, J. Guldner, and J. Shi, Sliding Mode Control in Electro-Mechanical Systems. CRC Press, 2009.

[13]. V. Utkin, "Variable structure systems with sliding modes," IEEE Transactions on Automatic Control, vol. 22, no. 2, pp. 212-222, Apr 1977.

[14]. H. Sira-Ramirez, "A geometric approach to pulse-width modulated control in nonlinear dynamical systems," IEEE Transactions on Automatic Control, vol. 34, no. 2, pp. 184-187, Feb 1989.

[15]. D. Liberzon, "Hybrid feedback stabilization of systems with quantized signals," Automatica, vol. 39, no. 9, pp. 1543-1554, 2003. 